\title{
Saphenous Vein Graft Aneurysm Late After Coronary Artery Bypass Grafting
}

\author{
Hiroko Kobayakawa, MD, Kazuaki Tanabe, MD, PhD, \\ Kazuto Yamaguchi, BS, Toshiaki Sumida, RMS, Junichi Kawai, PhD, \\ Toshiko Konda, RMS, Yoko Fujii, RMS, Toshikazu Yagi, RMS, \\ Atsushi Yamamuro, MD, Tomoko Tani, MD, Shigefumi Morioka, MD, PhD \\ and Yasuki Kihara, MD, PhD \\ Division of Clinical Laboratory and Division of Cardiology, Kobe General Hospital, Kobe, Japan
}

\begin{abstract}
Described is a rare case of a saphenous vein bypass graft aneurysm late after surgery. Doppler echocardiography was useful in assessing the inflow and outflow patterns of the aneurysm and its complications.
\end{abstract}

(J Echocardiogr 2006; 4: 86-88)

Key words: echocardiography, Doppler, saphenous vein bypass graft, aneurysm

A 77-year-old woman was admitted to our hospital for evaluation of ischemic heart disease. She underwent two coronary artery bypass graft (CABG) at ages 60 and 67 . With the first CABG in 1985, saphenous vein grafts (SVGs) were anastomosed to the left anterior descending (LAD), the obtuse marginal, and the posterior descending arteries (PD). She was asymptomatic until 1992 when she underwent coronary angiography for recurrent chest pain. The angiographic study demonstrated a tight stenosis of the SVG anastomosed to the LAD. The other two SVGs anastomosed to the obtuse marginal and the PD were occluded. She underwent subsequent $\mathrm{CABG}$ with three new bypass grafts - a left internal thoracic artery graft (LITA) to LAD, a SVG to the obtuse marginal artery, and the gastroepiploic artery (GEA) to the PD. However postoperative angiography showed that the LITA and GEA were occluded. She therefore underwent percuta-

Received April 21, 2006; revision received July 13, 2006; accepted August 10, 2006

Address for correspondence: Kazuaki Tanabe, MD, PhD

Division of Cardiology, Kobe General Hospital,

4-6 Minatojima-nakamachi, Chuo-ku, Kobe 650-0046, Japan.

Telephone: +81-78-302-4321

Fax: +81-78-302-2487

E-mail: ktanabe@leto.eonet.ne.jp

(C) 2006 Japanese Society of Echocardiography neous transluminal coronary angioplasty (PTCA) to the first SVG graft anastomosed to the LAD. The angiographic study status post PTCA demonstrated an intact SVG graft. The patient did well and had no chest pain for almost 10 years; she had been clinically man-

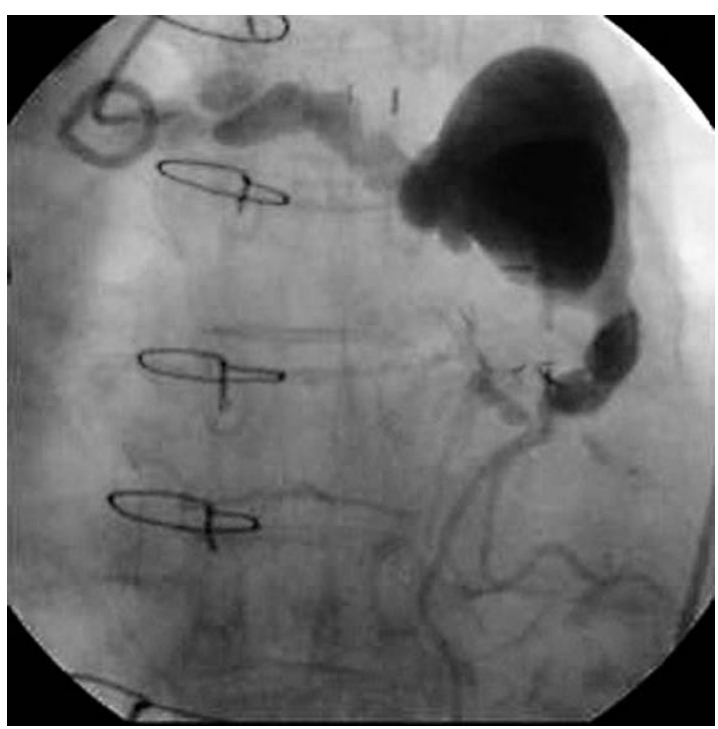

Fig. 1. Coronary angiography demonstrated a spindle-shaped aneurysm originating from the saphenous vein graft to the left anterior descending artery. 
aged by her family practitioner.

At this admission, an electrocardiogram did not show any ischemic changes, and cardiac enzymes were normal. A chest roenterogram showed a left hilar mass and dilatation of the cardiothoracic ratio and the thoracic descending aorta. Computed tomographic scan demonstrated a giant mass (diameter $50 \mathrm{~mm}$ ) adjacent to the left side of the main pulmonary artery. CAG demonstrated a spindle-shaped aneurysm originating from the SVG to the LAD (Figure 1) at the same
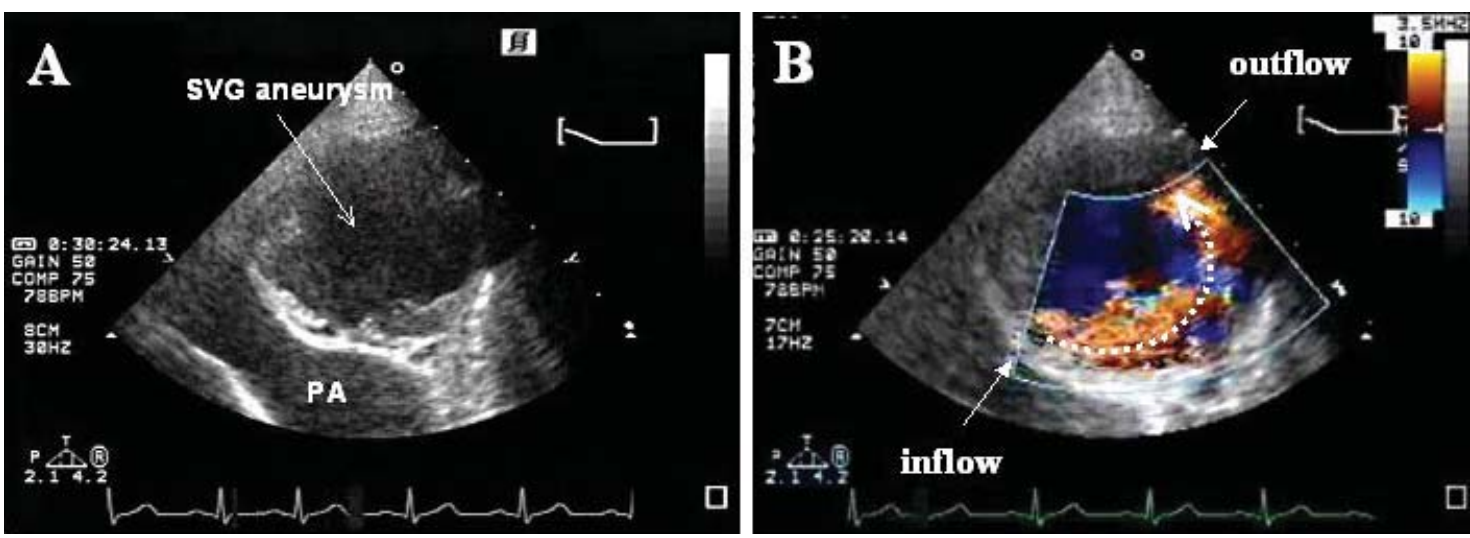

Fig. 2. A parasternal short axis view revealing a giant aneurysm with a mural thrombus, adjacent to the main pulmonary artery (PA) (A). Color Doppler echocardiography showed the inflow and the outflow of this aneurysm (B).
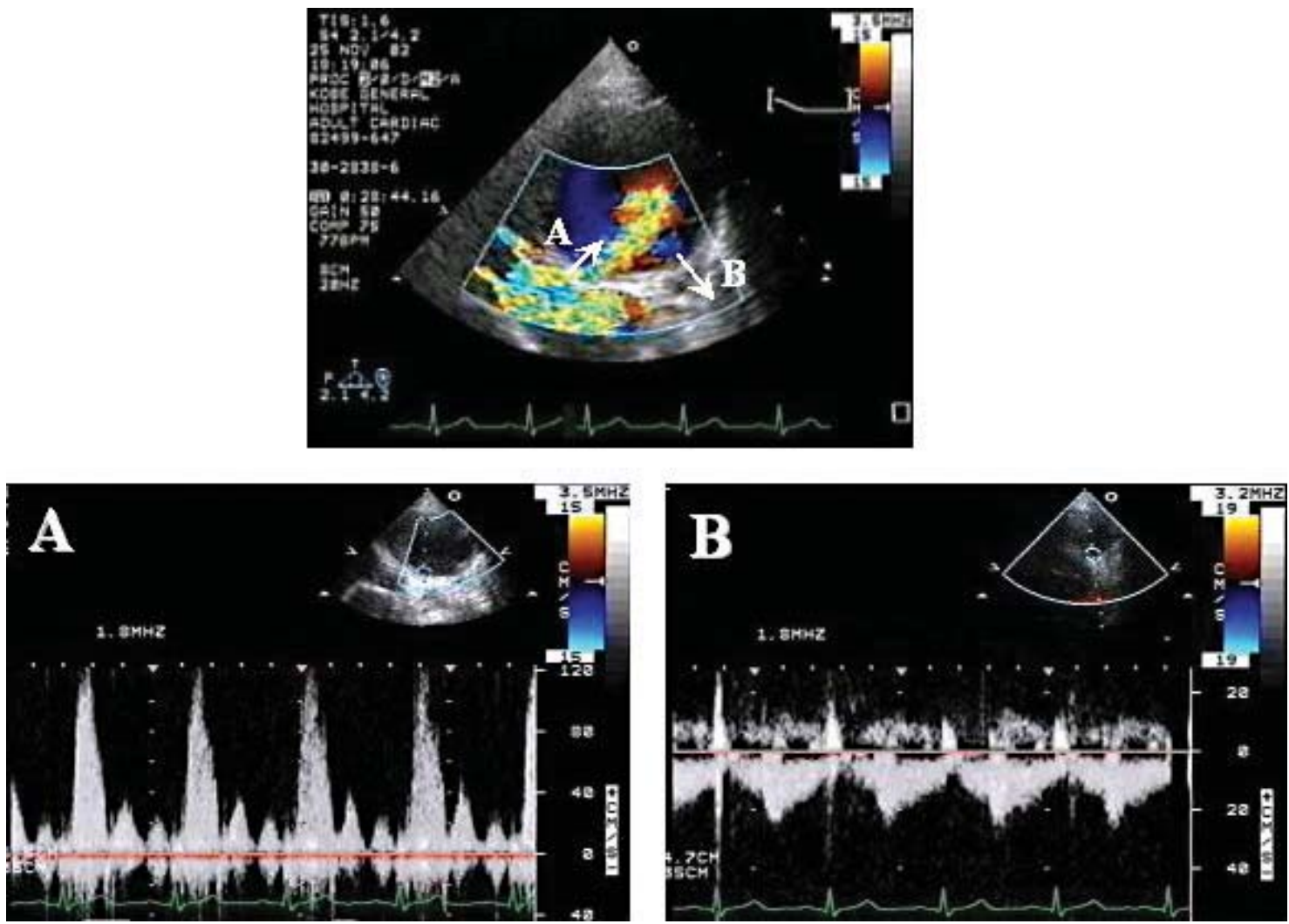

Fig. 3. The inflow to the aneurysm was an arterial blood flow pattern with a dominant systolic flow $(120 \mathrm{~cm} / \mathrm{s})(\mathrm{A})$. The outflow of this aneurysm had a coronary blood flow pattern with a dominant diastolic flow $(24 \mathrm{~cm} / \mathrm{s})(B)$ 
site where the PTCA was done.

Two-dimensional transthoracic echocardiography showed this aneurysm with a mural thrombus adjacent to the main pulmonary artery (Figure 2A). Color Doppler echocardiography showed the inflow and outflow of this aneurysm (Figure 2B). The inflow to the aneurysm had an arterial blood flow pattern with a dominant systolic flow $(120 \mathrm{~cm} / \mathrm{s})$ (Figure $3 \mathrm{~A})$. The outflow had a coronary blood flow pattern with a dominant diastolic flow $(24 \mathrm{~cm} / \mathrm{s})$ (Figure $3 \mathrm{~B})$, consistent with the patent graft flow pattern of the LITA assessed by transthoracic Doppler echocardiography [1]. Intravenous contrast echocardiography with Levovist revealed no communication between the SVG aneurysm and adjacent chambers or pulmonary artery. Resection of the aneurysm with revascularization was recommended; however the patient and her family elected conservative therapy because of her age and physical condition. She suddenly died soon after. The cause of the death remained unclear, as an autopsy was not performed.

SVG aneurysms late after CABG are rare complications. True aneurysms of SVG are atherosclerotic in nature and appear as a late postoperative complication more than five years after CABG [2]. In this case, occurence of the giant aneurysm may have been related to the previous PTCA. However, the CAG finding 5 years after PTCA for SVG demonstrated that the SVG remained intact, thus we believed the mass was not a pseudo but a true aneurysm. Previous reports describe SVG aneurysms to be associated with compression of the right atrium, non-Q-wave myocardial infarction [3], the development of a fistula between the graft and the right atrium [4-7], and superior vena cava obstruction [8]. The complications of an SVG aneurysm include thrombosis, embolism, and rupture. Most SVG aneurysms have been treated with either exclusion or resection of the aneurysms and, if neces- sary, with revascularization $[2,4,7]$.

In this case, no such findings were made, and it was clear that a fistula between the aneurysm and PA did not exist by transthoracic Doppler echocardiography. Doppler echocardiographic study was useful in assessing SVG patency and complications of the SVG aneurysm.

\section{References}

1. Takagi T, Yoshikawa J, Yoshida K, Akasaka T. Noninvasive assessment of left internal mammary artery graft patency using duplex Doppler Echocardiography from supraclaviclar fossa. JACC 1993; 22: 1647-52.

2. H Le Breton, D Pavin, T Langanay, et al. Aneurysms and pseudoaneurysms of saphenous vein coronary artery bypass grafts. Heart 1998; 79: 505-8.

3. Sahouri SJ, Steele RL. Aneurysm of saphenous vein graft to coronary artery presenting as non-Q-wave myocardial infarction secondary to mass effect. Cathet Cardiovasc Diagn 1995; 34: 325-8.

4. Riahi M, Stone KS, Hanni CL, et al. Right ventricularsaphenous vein graft fistula. Unusual complication of aortacoronary bypass grafting. J Thorac Cardiovasc Surg 1984; 87: 626-8.

5. Jukema JW, van Dijkman PRM, van der Wall EE. Pseudoaneurysm of a saphenous vein coronary artery bypass graft with a fistula draining into the right atrium. Am Heart J 1992; 124: 1397-9.

6. Zely P, Delarche N, Estrade G, et al. Double aneurysm of a venous aorto-coronary bypass graft. Arch Mal Coeur 1996; 89: 1213-6.

7. Yoshiharu Nishimura, Yoshitake Okamura, Takeshi Hiramatsu, et al. Hemoptysis caused by saphenous vein graft aneurysm late after coronary artery bypass grafting. J Thorac Cardiovasc Surg 2005; 129: 1432-3.

8. Eoin C. Kavanagh, Gormlaith Hargaden, Fidelma Flanagan, et al. CT of a Ruptured Vein Graft Pseudoaneurysm: an unusual cause of superior vena cava obstruction. AJR 2004; 183: 1239-40. 